\title{
Efficient Channel Access Techniques for Next Generation Mobile Networks
}

\author{
P. Bonus Reddy \\ Research Scholar, Dept. of CSE, \\ Mewar University, Rajasthan, India
}

\author{
P. Venkata Krishna, PhD \\ Assistant Professor, SPMVV, \\ Andhra Pradesh, India
}

\begin{abstract}
Attributed to the explosion in the number of mobile users, an imposition on the limits of bandwidth emerged that deliberately affects the network performance. Further, because of increasing applications it is essential to assure bandwidth and low delay. Existing Congestion Avoidance routing protocols select path guaranteeing delay and bandwidth. However, there are channel variations through which it fails to pass the information between the nodes. To overcome these concerns, we have proposed a crosslayer TDMA-based routing protocol to meet delay and bandwidth requirements. Further to cope with issues such as reliability and channel accessibility we have integrated AODV protocol along with the cross-layer TDMA. Also, to reduce cost and packet loss we have incorporated multipath routing methods.
\end{abstract}

\section{Keywords}

Channel Access Methods, TDMA, Cross-layer TDMA, AODV, Routing, Multipath routing.

\section{INTRODUCTION}

The rise of advanced technologies in telecommunications and computer networks has led to an increase in the number of mobile users that induces an upsurge of practice of wireless networks that extensively provides significant amount of advantages relatively to the wired systems. Wireless networks deliberately allow mobile communications, they do not entail more outlay that is obligatory in the maintenance of the wired systems, and they facilitate broadcasting in the expected manner. The wireless devices follow the IEEE 802.11 standard having varieties namely 802.11a, 802.11b and 802.11g, distinguished with the operational frequencies [1].Channel access, also known as 'multiple access' is an imperative facet that enables network stations share the volume of media by means of a multipoint transmission channel such as bus, hub, wireless and ring networks. Channel access has been established on a multiplexing technique that lets numerous data packets and signals to share a communication channel. Also, channel access is contingent on a multiple access protocol along with a control technique called Medium Access Control (MAC).

Channel access techniques are essentially classified under various appellations and packet multiplexing systems. Channel partitioning is fixed multiplexing via Frequency Division Multiple Access (FDMA), Time Division Multiple Access (TDMA), and Code Division Multiple Access (CDMA)methods in wireless mobile networks [2].These methods are conflict-free and the allocation taking place is static. The methods that are dynamically allocated include Polling and token passing [3]. The static methods entail partitioning the channel into time slots or frequency slots where every slot is provided to a node for limited usage. However, there are certain downsides of these methods for basing on the varying number of users that instigate inefficiency. Likewise, in the event of the users being inactive, it causes the slots to be unoccupied there by it remains inoperative [4]. Contrariwise, relative to FDMA, CDMA and certain contention based channel access techniques such as ALOHA, CSMA and Binary Tree, TDMA based channel access technique has better energy efficiency and is collision-free [5].Therefore for the purpose of this research, we have employed the TDMA technique.

\subsection{TDMA}

A channel access technique,TDMA is usually employed for shared medium networks. This technique lets numerous users use the same frequency channel through signal partitions into many time slots. The transfer ensues in a speedy sequence, wherein the users sequentially transfer at their own time slot. Consequently, the multiple stations are able to use the same channel, at the same time use simply a fragment of its channel capacity. TDMA has been extensively employed satellite systems and combat-net radio systems besides the digital $2 \mathrm{G}$ cellular systems including Global System for Mobile communications, IS136, Personal Digital Cellular (PDC) and iDEN, and in the Digital Enhanced Cordless Telecommunications (DECT) standard for mobile phones.

TDMA aids in allowing several earth stations to communicate sporadically having alike frequency. However due to the partitioned time slots for the transmission, there is no intersection when the transmitted entity reaches the satellite in order, hence effectively collected. The TDMA process, having a frame structure for the provision of the precise time information, will establish an out link control for each of the remote sites comprising of certain control data. In this way, all the remote sites are informed the time of using the slot intermittently. The transmission time is perhaps static hence the allocation of the entire TDMA time frame will be proportional while the allotted time slot is attuned according to the requirement of the traffic of each site [6].

The frame structure of TDMA is depicted in Figure 1. It is evident from the figure that a data stream is apportioned into several frames that are further apportioned into time slots. In TDMA, there are many transmitters which are connected to a receiver. However, for a mobile phone it is tedious to establish a transmission route from the mobile station to the base station, attributed to the mobility of the device that results in variation of the time that was supposedly intended to correspond the transmission between the nodes. 


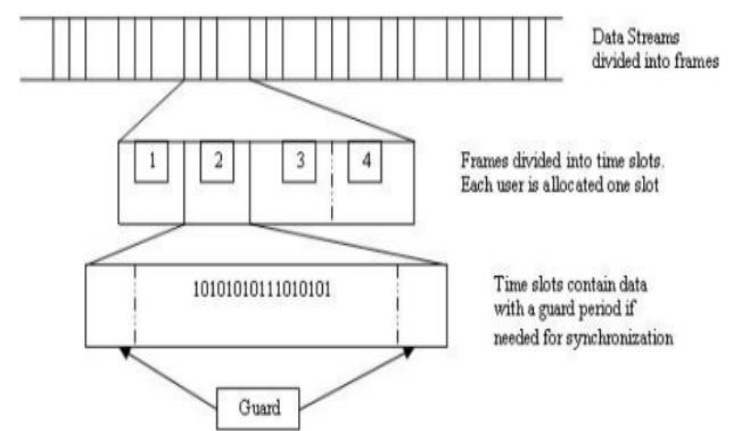

Figure 1: Frame structure of TDMA

The characteristics of TDMA are as below:

- $\quad$ TDMA uses a single carrier frequency together with manifold users

- Due to the intermittent transmission, the handoff is easier

- In the event of more demand, the slots are allocated dynamically

- There is a smaller amount of power control relative to CDMA because of decreased intracell interference

- There is a high synchronization operating cost relative to CDMA

- Innovative equalization is obligatory in case of excessive data rates and the channel is discerning about the frequency, establishing Inter-symbol interference

- Employing the resources from neighboring cells is complex

- There is a complication with regard to assigning frequency/slot

Since TDMA is capable of being dynamic characteristically in the event of more demand, an algorithm that will schedule dynamically, keeps a variable number of time slots for all the frames to variable bit-rate data streams. The various applications of a dynamic TDMA include HIPERLAN/2 broadband radio access network, IEEE 802.16a WiMax, Bluetooth, Packet Radio Multiple Access (PRMA) technique for collective circuit switched voice interface and packet data, TD-SCDMA, ITU-T [6].

\section{RELATED STUDIES}

Sanjiv Tokekar et al [7] proposed a novel fixed channel allocation method for the purpose of channel allocation in a classified GSM network. The authors carried out their work in mainly two steps; the foremost step employed atypical fixed channel allocation for allocating same number of channels to all the cells and at that juncture reallocating the redundant channels having lesser traffic cells amid the neighbors in a statically. Every Normally GSM operators begin their services with a fewer base transceiver stations. However, the number of stations is amplified accordingly to the traffic. In general, there is disparity in the size of cells and several choices pertaining to allocation are rational yon the amassed data. The proposed system was considered ideal for the aforementioned circumstances.
An efficient bandwidth reservation technique centered on AODV multi-path routing for hybrid networks was proposed by [8]. Hash Table-Based Node Identification (HTNI) technique to easily indicate user movement that makes it convenient to detect many routes. It employed reservation of bandwidths for many flows in these networks along with a demarcation of QoS based bandwidth factor, QFRB, which was made for the purpose of reserving the bandwidth by discerning the diversity of the flows. The authors developed an ideal QoS architecture for the cross-layer model. This technique was commendably evaluated and substantiated on the basis of performance metrics including packet throughput and endto-end packet delay that were matched in two cases i.e. with reservation and the other without reservation.

A study [9] pertinent to energy efficient low duty cycle MAC protocol for Wireless Body Area Networks accentuates the comparison of TDMA protocol with CSMA/CA. Since TDMA is collision-free, has resilience to faults arising during the transmission, is more energyefficient, the study delineated that TDMA is better than CSMA/CA protocol. Conversely, the authors outlined the higher synchronization costs in TDMA and due to increasing data requirements that instigates queues, the energy-efficiency of TDMA may be lowered which may also be affected because of varying network topology.

Another research [10] employing anin-vivo communications between mobile nodes in Body Sensor Networks with an energy efficient TDMA protocol for uplink/downlink asymmetric network architecture described that the lowering the time frame for the data slots using the scheduling method in dynamic TDMA and the variable frame arrangements gradually enhanced the performance. Similarly, [11] demarcated the energyefficient TDMA protocol which accordingly in this research aided in decreasing the synchronization costs of TDMA by reducing the "idle listening" in the sleep mode. The notion was to intermittently listen to the synchronization messages rather than a continual listening that causes less power consumption. The limitation of this research was that there was absolutely no wake-up radio technique in the event of higher traffic demand. On the contrary, the research [12] that presented a wake-up radio MAC protocol, was evidently energy efficient as the node went on to sleep mode at the time of no transmission instead of staying idle, and using a wake-up radio method, would wake up in times of demand.

An improved MAC protocol [13]emphasizing on simplicity, reliability and power effectiveness was employed during the contention access phase for command packets and best effort data packets. While CSMA/CA was employed during the contention free phase which comprised of data transmission only. However, this protocol has more delay relative to TDMA and FDMA.

The use of Orthogonal FDMA for multiple access in wireless networks is restrained. FARA [14], in which an orthogonal FDMA wherein the transmission route is from a cell site to the mobile phone, having a per-subcarrier rate adaptation. In this system there is a single transmitter due to which the symbol configuration was not contemplated to be a concern. Further in [15] and [16], the application of orthogonal FDMA was simply as a synchronized channel access protocol where the nodes control one bit of ACK info on various subcarriers subsequent to obtaining a broadcast frame. 


\section{FORMULATION OF THE PROBLEM}

In the wireless mobile networks, the massive and unremitting growth of users causes a constriction on bandwidth. It is extremely essential to have optimum bandwidth utilization in order to provide service to all the users. Further the channel accessibility instigates inefficient performance of the networks and there exist several defies that have been snowballing due to technological impingements and the needs for optimization [17].Also, attributed to the cumulative growth of real-time and multimedia applications, it is immensely required to develop a system that provides higher bandwidth and no delay.

Also, besides the limitation of bandwidth allocation, channel accessibility and delay cause poor performance of networks. Attributable to channel variations, the transmission of information between the nodes is also affected. These issues in the networks cause lack of reliability, therefore it is essential to develop a channel access method which efficiently copes with the aforementioned concerns.

\section{EVALUATION PARAMETERS 4.1 Path Loss}

The path loss is the local average received signal power at the receiver node compared with the transmission power of the transmission node. The path loss is given as below:

$$
L=20 \log _{10} \frac{4 \pi d}{\lambda}
$$

Where $\mathrm{L}$ is the path loss, $\mathrm{d}$ is the distance, and $\lambda$ is the wavelength [18].

\subsection{Cost}

The route cost calculation of a link amid two nodes is the resultant from the free space path loss of radio waves. The cost is contingent on the number of nodes employed, the power consumption and path loss. The cost is given by:

$$
C=a * L
$$

Where $a$ is a constant. The total route cost is computed by adding the cost at each node.

\subsection{Network Throughput}

The network throughput $\mathrm{T}$ is the mean number of effectively transmitted bits per Sec-Hz-unit-area, depicted as

$$
T=\lambda_{b} P_{a}\left(1-P_{O}\right) R_{O}
$$

Where $\lambda_{b} P_{a}$ is the base station density and $R_{O} \triangleq$ $\log _{2}(1+\hat{\gamma}), P_{O}=P_{r}(S \leq \hat{\gamma}), \hat{\gamma}$ is the given threshold, and $\mathrm{S}$ is the receive signal-to-interference along with the noise ratio.

\subsection{End-To-End Delay}

The end-to-end delay in a wireless network is the amount of queuing and transmission delays at source and transitional nodes. It is depicted as,

$$
E[D]=\frac{1}{N(N-1)} \sum_{\forall(S, D)} \sum_{\begin{array}{c}
\forall \text { link }(i, j) \text { in path } \\
\text { selected to route }(S, D)
\end{array}} E\left[D_{i j}\right]
$$

\section{PROPOSED SYSTEM}

For the purpose of this research, we have employed a cross-layer TDMA protocol to overcome channel variations, limited bandwidth and delay. However, by adding AODV protocol, we were able to ease the disputes with regard to channel accessibility, thus obtaining the reliability of the system. Further, complementing the system with multipath routing protocol, the costineffectiveness and packet loss was handled. Hence a comprehensive study of these three protocols is delineated.

\subsection{Cross-Layer TDMA Protocol}

We have proposed a cross-layer TDMA based reactive routing protocol from [19] that will offer bandwidth and delay. Mainly, the notion behind this protocol is optimization of the network throughput. This is achieved by reducing the number of neighbors connected with the routes. Meanwhile, in any wireless network, the time slot utilized by one node is not available for the usage of the neighbors of that node for data transmission. Therefore, choosing a route with lesser number of neighbors leads to expediency. Further, for the purpose of assigning the collision-free channel in TDMA, the access time will be partitioned into super frames that are subdivided into control and data time slots. All the nodes are allotted a control time slot that is used by the nodes for transmission of the control information, while the remaining super frame is intended for transmission of data. These nodes are essentially required to contest for the sake of reserving the time slots. A time slot $s$ is counted as free and possibly will be allotted to send the data from a node $A$ to a node $B$ on the event of the below prerequisites[20]:

- Slot $s$ is unscheduled for transmission in both nodes

- The slot is unscheduled for reception in a node $C$ that is in fact a single hop neighbor of $A$

- The slot is unscheduled for sending in the node $C$ that is in fact a single hop neighbor of $B$

However, at the time of the allocation of the slots on a link $(\mathrm{x}, \mathrm{y})$, the single hop neighbors are unable to utilize them because some interferences are likely to ensue. The allocation of the slot is directly proportional to the number of neighbors since the allocation influence is determined by this number. This influence indicates the evasion of the ability of the nodes to transmit data except the flow $f$ packets as the allocation of the time slots support a flow $f$.

The assignment of slots effects two subdivisions of the nodes i.e. nodes passing the data packet of the new flow further thereby establishing a new route, and their neighbor nodes. Thus, the prevailing significance is to decrease the amount of hops in one path and pick out the nodes in a way that the number of affected neighbors is less. The sum of time slots assigned to flows is depicted by theorem 1 [19].

Theorem 1: given a flow with $k$-slot requirements passed on by a path $P=\left\langle v_{1}, \ldots, v_{N}\right\rangle$, the number of time slots assigned to that flow is $S A(P)=2 k(N-1)$.

While any node reserves bandwidth, if the amount of neighbor nodes is high, the network throughput is low. Therefore, the QoS-based routing protocols are required to elect paths in which there is minimum affect.

Theorem 2 provides the number of slots that are reserved by a flow $f$. 
Theorem 2: given a flow with $k$-slot necessities passed on by a path $P=\langle v 1, \ldots, v N\rangle$, the flow effect on the neighborhood of path $P$, denoted $S R(P)$, is :

$$
S R(P) \leq k\left(N_{1}-1\right)+\sum_{i=2}^{n-1} 2 k\left(N_{i}-1\right)+k\left(N_{n}-1\right)
$$

where $\mathrm{N}_{\mathrm{i}}$ is the number of neighbors of node $\mathrm{v}_{\mathrm{i}}$. The following lemma 1 illustrates the effect of time slot allocation fora flow which is resulting from the theorems 1 and 2. It also gives abound on the number of slots affected by a flow $f$.

Lemma 1: given a flow with $k$-slot requisites passed on by a path $P=\langle v 1, \ldots, v N>$, the flow effect on the complete network, represented $S I(P)$, is:

$$
S I(P) \leq k N_{1}+\sum_{i=2}^{n-1} 2 k N_{i}+k N_{n}
$$

The routing issue regarded in this paper is a multiconstricted idealproblem which is NP-complete [20]. By employing the above lemma, the problem is overcome as follows:

Given a source sand destination $d$, the best routing protocol is that which returns a path $P \in \pi(s, d)$ such that $P$ meets bandwidth anddelay requirements and $\forall P^{\prime} \in \pi(s, d)$ $\Rightarrow S I(P) \leq S I\left(P^{\prime}\right) . \pi(s, d)$ is the set of path between $s$ and $d$.

The path efficiencyis computed from the affected bandwidth, $B I(P)$, indicating inaccessible bandwidth due to the affect of the slot assignement:

$$
B I(P)=\frac{1}{T} S I(P) T_{S} C
$$

where $T$ is the TDMA super frame period, $T_{s}$ the slot length and $C$ the link capacity.

\subsection{AODV Protocol}

The Ad-hoc On-demand Distance Vector (AODV) routing protocol [22][23] is also a reactive routing protocol wherein the path is ascertained as per the demand. The message interactions in an AODV protocol is depicted in Figure 2.In this protocol, the Hello messages are employed to determine and observe the links to neighbors, and in such cases, all the nodes that will intermittently broadcast this message to their neighbors. In the event of a failure of a node receiving a hello message, the link is broken.

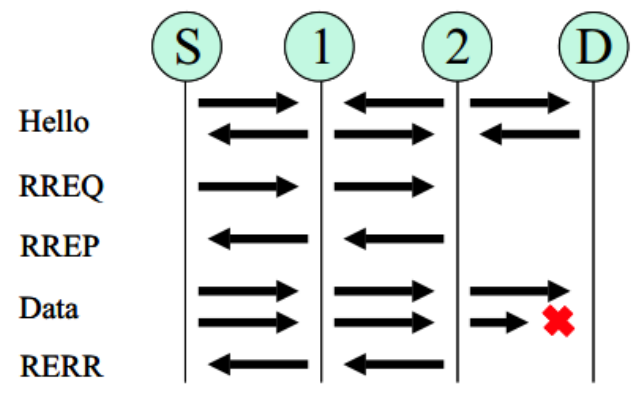

Figure 2: Protocol Messaging in AODV

At the time of data transmission to some node, the sending node will broadcast a Route Request (RREQ) for the receiving node. A route to the source will be established when the nodes in the midway receive this packet. In case, the node which receives the packet has not received the packet earlier, then it is contemplated to be an intermittent node and not the final node. The packet is broadcasted again. On the other hand, if the receiving node is the final node, then a Route Replay (RREP) message is instigated. This message is characteristically unicast in a 'one hop after the other' manner to the initial node. When this node is broadcasted, the route to the final node is made. If the source receives this message, then the route to the destination is logged and the data is sent to the final node. In the event of multiple RREP messages, the source will pick the shortest hop count. When the data passes to the destination, all the intermittent nodes in the route will update their timers, upholding the routes in the routing table. Among all the routes, if one route has not been utilized for a specific amount of time, the nodes are uncertain of the availability of that route, deleting it from the routing table. As it was, during the transmission, if there is a link break, a Route Error (RERR) is transmitted to the initial node hop-by-hop. During this transmission, all the intermittent nodes will cancel the routes to the destination. However, the initial node also cancels the route and restarts the entire process [24].

\subsection{Multipath Routing Algorithm}

Multipath routing algorithm makes use of the fundamental physical network resources by employing multiple sourcedestination routes (See Figure 3). This type of routing has been employed for several uses such as bandwidth aggregation, reducing end-to-end delay, escalatingfaulttolerance, and augmenting reliability and load balancing. Multipath routing has three phases namely path discovery, traffic dispersion and path maintenance [25].

Path discovery is the method of ascertaining the paths that can be availed for creating source-destination pairs. While the algorithm has many conditions to employ for selecting a subset of paths in the discovery process.

For dispersing traffic, several approaches for assigning traffic in the obtained paths exist. The algorithm is accountable for opting to send the traffic by means of an ideal path, while the other paths are held as a reserve. Otherwise, all the obtained paths could possibly be employed simultaneously. The algorithm comprises a method for selecting the path based on certain specifics such a shop-count. Contrariwise, the QoS routing accounts for selection of a subset of paths in the event of a joint evaluation parameter satiates the QoS conditions.

Maintaining the paths is an imperative aspect of the multipath routing algorithm because, after a certain period of time, the paths may be unavailable subject to link/node failures. Therefore, restoring those paths subsequent to the initial path discovery will be undertaken subsequent to the path failure.

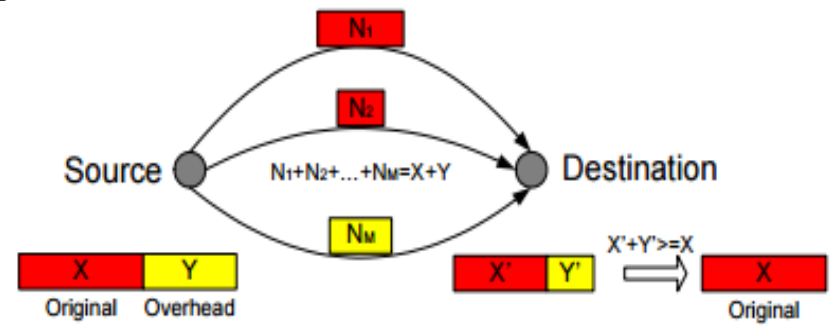

Figure 3: Multipath Routing 
Multipath routing offers fault tolerance and delay reduction by routing same information through the reserve paths. In the event of failure, this algorithm is effectively reliable. Further load balancing is achieved as the overworked/congested routes can be switched with the reserve routes. Bandwidth aggregation is another aspect obtained through multipath routing wherein the data is split into several streams and each stream is sent via dissimilar routes.

Furthermore, to optimize this system, we have incorporated the aspects of Cuckoo Search Algorithm (CS) and improved Genetic Algorithm (GA).The CS and GA distinctly are population-based algorithms, and the hybrid CS-GA similarly is a population-based algorithm, employing a population of answers to continue to the global answer.

In order to disperse the preliminary solution during the solution space, the foremost population will be randomly produced, which remains constant throughout the whole process. In every phase of population generation wherein the algorithm mentioned in Figure 4 produces new population that have enhanced points through the GA ideologies, it is required to primarily experience crossover followed by mutation. Subsequent to every process, certain procedure for maintaining exclusivity is executed to pick out the optimum solutions. Further in the existing population, the obtained optimum solution ahead of the completion of the generation will implement Levy flight in order to improve the assessment of the solution space for the next generation. The crossover and mutation process that is reiterated steers en route for the ideal most solution.

The notion of the CS algorithm is that a cuckoo lays egg one after the other executing the Levy flights. However here the perception is that, two cuckoos will procreate two cuckoo eggs. Since the cuckoo lays eggs in another bird's nest, the cuckoo will impersonate the eggs of the bird whose nest is used. For constituting this comportment, adding a mutation operator was ideal, as it reveals the behavior of the gene mutation of the cuckoo eggs' for improving the yield. Despite gene mutation, the nest's bird might detect that the egg is foreign, basically due to the egg quality. Hence, exclusiveness will be undertaken, thus the cuckoo bird will throw out the other eggs and keep only hers [26].

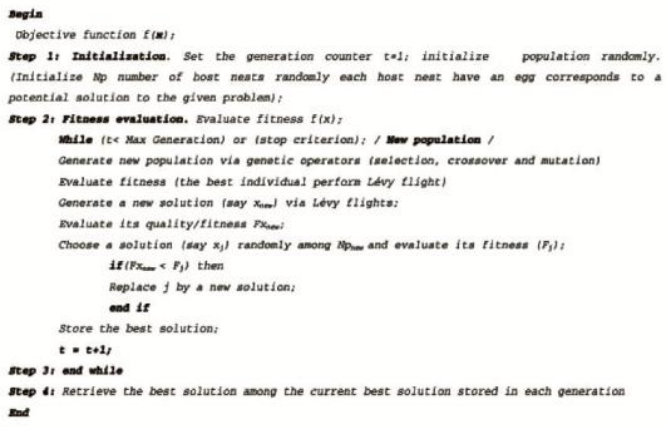

Figure 4: Hybrid CS-GA algorithm

\section{SIMULATION AND RESULTS}

In this section, we will assess the performance of the proposed system and provide a comprehension on the impact on the evaluation parameters. The simulation was carried out in MATLAB. For the purpose of this study, we have considered 50 iterations for successful transmission.
The initial population is generated randomly based on the bandwidth limits i.e. the minimum and maximum values of bandwidth of the network. The following equation is employed for the random generation:

$$
I=\min \_v a l+\left(\max \_v a l-\min \_v a l\right) * r a n
$$

The initial size (I) depends on the population size given in paper. We selected an ideal value from the fitness function and the initial random values are updated by integrating the algorithm parameters i.e. the arithmetic crossover and mutation by including the Levy flight distribution. An optimum value is selected by iterating the process.

As seen in Figure 5, the path loss significantly reduced at the $19^{\text {th }}$ iteration and remained constant there on. Similarly in Figure 6, the cost of the system was observed to have a drastic reduction at the $5^{\text {th }}$ iteration.

Figure 5: Path Loss

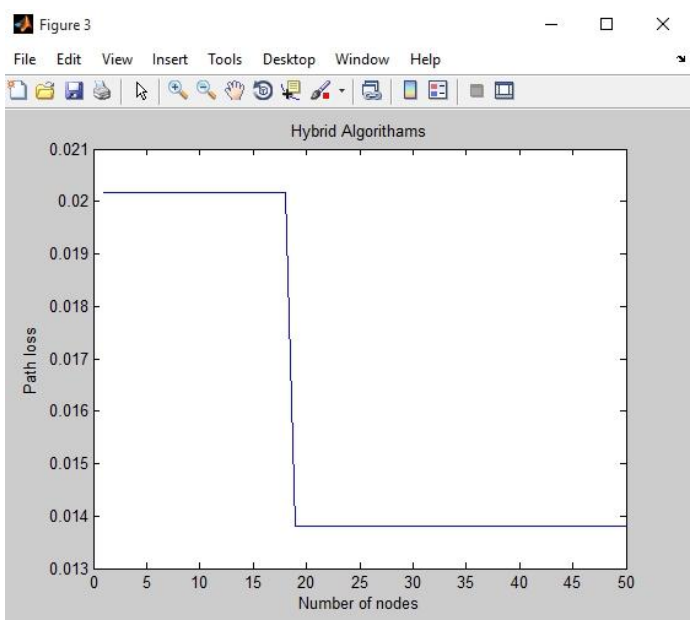

Figure 6: Cost

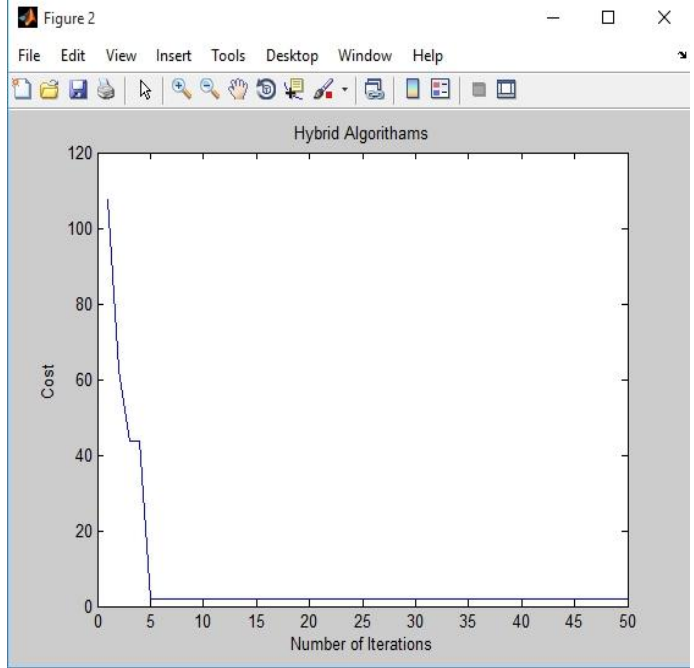

Figure 7 indicates the network throughput by comparing the proposed system with the QoS routing. The network throughput in our system was considerably higher than the network throughput obtained in the QoS system. Lastly, the end-to-end delay in the Figure 8 gradually increased for the multi-hop transmission from the $2^{\text {nd }}$ iteration and remained constant from the $9^{\text {th }}$ iteration. 
Figure 7: Network Throughput

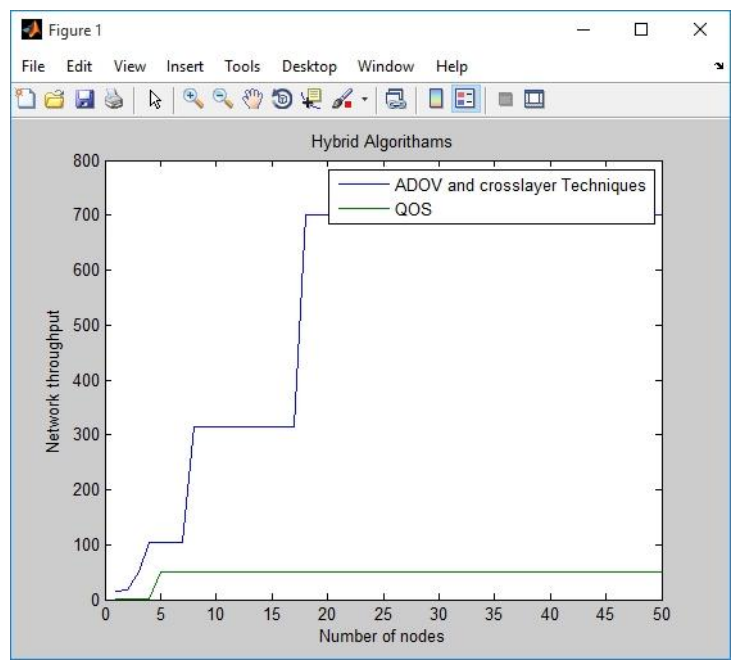

Figure 8: End-to-End Delay

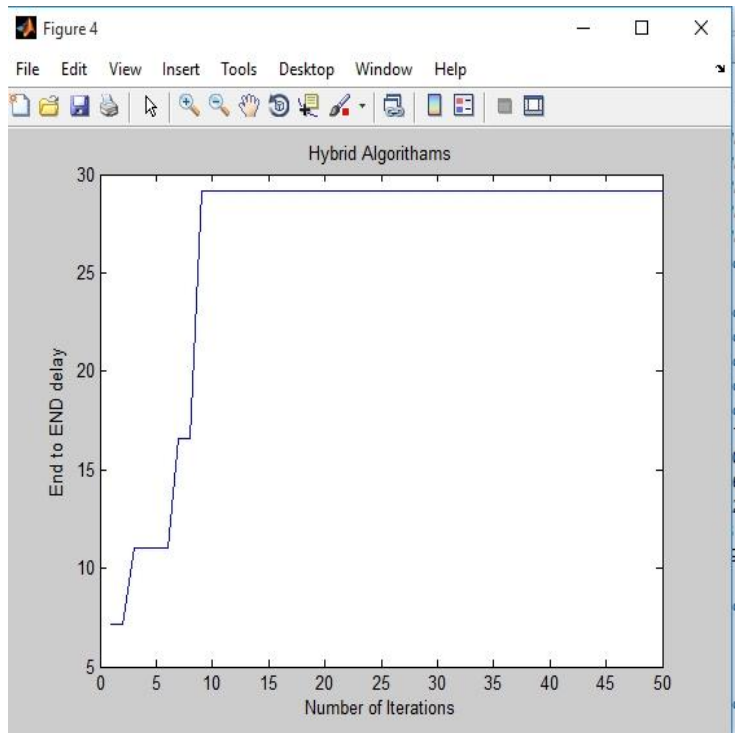

\section{CONCLUSION}

A novel technique collectively using cross layer TDMA based bandwidth reservation scheme, AODV routing and multipath routing was proposed to overcome issues pertaining to bandwidth, cost, delay, channel variations, accessibility, path loss and reliability. The performance metrics like network throughput, end-to-end delay, cost and path loss were used to measure the effectiveness of the system and it was found that the system is better than the QoS techniques. By including more metrics to ascertain the performance of the system, we can further improve the system.

\section{REFERENCES}

[1] R. Sharma, D. G. Singh, and R. Agnihotri, "Comparison of performance analysis of 802.11a, $802.11 \mathrm{~b}$ and $802.11 \mathrm{~g}$ standard," (IJCSE) International Journal on Computer Science and Engineering, vol. 02, pp. 2042 - 2046, 2010.

[2] J. F. Kurose and K. W. Ross, Computer Networking: A Top-Down Approach, 5th ed. Addison - Wesley, 2010.
[3] Er Liu, Multiple Access Methods Multiple Access Methods, Helsinki University of Technology Helsinki University of Technology S-72.333, Postgraduate Seminar on Radio Communications.

[4] P. S. Tomar and P. G. Poonacha, "An efficient channel access method using polling and dynamic priority assignment," 2013 4th International Conference on Computer and Communication Technology (ICCCT), Allahabad, 2013, pp. 145-150.

[5] I. Rhee, A. Warrier, J. Min, and L. Xu, "DRAND: distributed randomized TDMA scheduling for wireless ad-hoc networks," in Proceedings of the 7th ACM International Symposium on Mobile Ad Hoc Networking and Computing (MOBIHOC '06), pp. 190-201, May 2006.

[6] Rupesh Singh, Multiple Access Techniques For 4G Mobile Wireless Networks, International Journal of Engineering Research and Development, Volume 5, Issue 11 (February 2013), PP. 86-94

[7] SanjivTokekar, NeeteshPurohit, Analysis of a New Fixed Channel Allocation Scheme for a Sectorized GSM Cellular Network, Proc. of International Conference on Wireless and Optical Communications Networks (IFIP'06), 2006, pp.1-5.

[8] P. V. Krishna, N. C. S. N. Iyengar, S. Misra, An Efficient Hash Table-Based Node Identification Method for Bandwidth Reservation in Hybrid Cellular and Ad-Hoc Networks, Computer Communications (Elsevier), Vol. 31, No. 4, 2008, pp. 722-733

[9] Lin, L., Wong, K. J., Kumar, A., Tan, S. L., \&Phee, S. J. (2011, June). An energy efficient MAC protocol for mobile in-vivo body sensor networks. In Ubiquitous and Future Networks (ICUFN), 2011 Third International Conference on (pp. 95-100). IEEE.

[10] Yan, Z., \& Liu, B. (2011, July). A context aware MAC protocol for medical Wireless Body Area Network. In Wireless Communications and Mobile Computing Conference (IWCMC), 2011 7th International IEEE Conference, (pp. 2133-2138).

[11] Kwak, K. S., Ullah, S., \&Ullah, N. (2010, November). An overview of IEEE 802.15. 6 standard, In Applied Sciences in Biomedical and Communication Technologies (ISABEL), 2010 3rd International Symposium on (pp. 1-6). IEEE.

[12] Ameen, M. A., Ullah, N., \&Kwak, K. (2011, October). Design and analysis of a MAC protocol for wireless body area network using wakeup radio. In Communications and Information Technologies (ISCIT), 2011 11th International Symposium on (pp. 148-153). IEEE.

[13] NF Timmons and WG Scanlon. An adaptive energy efficient mac protocol for the medical body area network. In Wireless Communication, Vehicular Technology, Information Theory and Aerospace \& Electronic Systems Technology, 2009. Wireless VITAE 2009. $1^{\text {st }}$ International Conference on, pages 587593. IEEE, 2009 
[14] H. Rahul, F. Edalat, D. Katabi, and C. Sodini. Frequency-aware rate adaptation and mac protocols. In Mobicom, 2009.

[15] A. Dutta, D. Saha, D. Grunwald, and D. Sicker. SMACK: a smart acknowledgment scheme for broadcast messages in wireless networks. In SIGCOMM '09., New York, NY, USA, 2009. ACM.

[16] B.-S. Kim, S. W. Kum, and R. L. Ekl. Ofdma-based reliable multicasting mac protocol for wlans. IEEE Transactions on Vehicular Technology, 57(5), 2008.

[17] Mohammad Anbar and D P Vidyarthi, "On Demand Bandwidth Reservation for Real-Time Traffic in Cellular IP Network using Particle Swarm Optimization", International Journal of Business Data Communications and Networking, Volume 5, Issue 3, 53-66, July - September 2009.

[18] Anderson J. B., T. S.Rappaport, and S. Yoshida, (1995), Propagation measurement and models for wireless communication channels. Communications Magazine, IEEE 33.1, 42-49.

[19] David Espes, ZoubirMammeri, Delay and Bandwidth constrained Routing with Throughput optimization in TDMA-based MANETs, 2009 3rd International Conference on New Technologies, Mobility and Security, Cairo, 2009, pp. 1-5.

[20] W.H. Liao, Y.C. Tseng, K.P. Shih, “A TDMA-Based Bandwidth Reservation Protocol for QoS Routing in a
Wireless Mobile Ad Hoc Network", IEEE International Conference on Communications, 2002.

[21] R.K. Ahuja, T.L. Magnanti, J.B. Orlin, Network Flows: Theory, Algorithms, and Applications, Prentice Hall, Englewood Cliffs NJ, 1993.

[22] C. E. Perkins, E. M. Belding-Royer, and S. Das. Ad hoc On-Demand Distance Vector (AODV) Routing. RFC 3561, July 2003.

[23] C. E. Perkins and E. M. Royer. The Ad hoc OnDemand Distance Vector Protocol. In C. E. Perkins, editor, Ad hoc Networking, pages 173-219. AddisonWesley, 2000

[24] Ian D. Chakeres, Elizabeth M. Belding-Royer, AODV Routing Protocol Implementation Design, Proceedings of the 24th International Conference on Distributed Computing Systems Workshops - W7: EC (ICDCSW'04), p.698-703, March 23-24, 2004

[25] Jack Tsai and Tim Moors, A Review of Multipath Routing Protocols: From Wireless Ad Hoc to Mesh Networks, National ICT Australia (NICTA), University of New South Wales, Australia.

[26] G. Kanagaraj, S.G. Ponnambalam, N. Jawahar, A hybrid cuckoo search and genetic algorithm for reliability-redundancy allocation problems, Computers \& Industrial Engineering, Volume 66, Issue 4, December 2013, Pages 1115-1124. 